\title{
Probing ultrafast magnetic-field generation by current filamentation instability in femtosecond relativistic laser-matter interactions
}

\author{
G. Raj ${ }_{1}^{1, *, \dagger}$ O. Kononenko, ${ }^{1, *}{ }^{*}{ }^{\ddagger}$ M. F. Gilljohann, ${ }^{2,3}$ A. Doche,${ }^{1}$ X. Davoine, ${ }^{4}$ C. Caizergues, ${ }^{1}$ Y.-Y. Chang, ${ }^{5}$ \\ J. P. Couperus Cabada ${ }^{5},{ }^{5}$ A. Debus, ${ }^{5}$ H. Ding, ${ }^{2,3}$ M. Förster, ${ }^{2,3}$ J.-P. Goddet, ${ }^{1}$ T. Heinemann,,${ }^{6,7,8}$ T. Kluge, ${ }^{5}$ T. Kurz,,${ }^{5,9}$ \\ R. Pausch, ${ }^{5}$ P. Rousseau, ${ }^{1}$ P. San Miguel Claveria, ${ }^{1}$ S. Schöbel,,${ }^{5,9}$ A. Siciak, ${ }^{1}$ K. Steiniger, ${ }^{5}$ A. Tafzi, ${ }^{1}$ S. Yu, ${ }^{1}$ B. Hidding, ${ }^{7,8}$ \\ A. Martinez de la Ossa, ${ }^{6}$ A. Irman, ${ }^{5}$ S. Karsch, ${ }^{2,3}$ A. Döpp, ${ }^{2,3}$ U. Schramm, ${ }^{5,9}$ L. Gremillet, ${ }^{4}$ and S. Corde (1) ${ }^{1, \S}$ \\ ${ }^{1}$ LOA, ENSTA Paris, CNRS, Ecole Polytechnique, Institut Polytechnique de Paris, 91762 Palaiseau, France \\ ${ }^{2}$ Ludwig-Maximilians-Universität München, Am Coulombwall 1, 85748 Garching, Germany \\ ${ }^{3}$ Max Planck Institut für Quantenoptik, Hans-Kopfermann-Str. 1, Garching 85748, Germany \\ ${ }^{4}$ CEA, DAM, DIF, 91297 Arpajon, France \\ ${ }^{5}$ Helmholtz-Zentrum Dresden - Rossendorf, Institute of Radiation Physics, Bautzner Landstrasse 400, 01328 Dresden, Germany \\ ${ }^{6}$ Deutsches Elektronen-Synchrotron DESY, 22607 Hamburg, Germany \\ ${ }^{7}$ Scottish Universities Physics Alliance, Department of Physics, University of Strathclyde, Glasgow G4 ONG, United Kingdom \\ ${ }^{8}$ Cockcroft Institute, Sci-Tech Daresbury, Keckwick Lane, Daresbury, Cheshire WA4 4AD, United Kingdom \\ ${ }^{9}$ Technische Universität Dresden, 01062 Dresden, Germany
}

(Received 28 July 2019; revised manuscript received 22 December 2019; accepted 7 April 2020;

published 4 May 2020)

\begin{abstract}
The current filamentation instability is a key phenomenon underpinning various processes in astrophysics, laboratory laser-plasma, and beam-plasma experiments. Here we show that the ultrafast dynamics of this instability can be explored in the context of relativistic laser-solid interactions through deflectometry by low-emittance, highly relativistic electron bunches from a laser wakefield accelerator. We present experimental measurements of the femtosecond timescale generation of strong magnetic-field fluctuations, with a measured line-integrated $B$ field of $2.70 \pm 0.39 \mathrm{kT} \mu \mathrm{m}$. Three-dimensional, fully relativistic particle-in-cell simulations demonstrate that such fluctuations originate from the current filamentation instability arising at submicron scales around the irradiated target surface, and that they grow to amplitudes strong enough to broaden the angular distribution of the probe electron bunch a few tens of femtoseconds after the laser pulse maximum. Our results open a branch of physics experiments investigating the femtosecond dynamics of laser-driven plasma instabilities by means of synchronized, wakefield-accelerated electron beams.
\end{abstract}

DOI: 10.1103/PhysRevResearch.2.023123

\section{INTRODUCTION}

The Weibel-type current filamentation instability (CFI) $[1,2]$ has been extensively investigated in past decades owing to its recognized importance in an increasing variety of plasma environments. Induced by temperature anisotropies or relative drifts between the plasma constituents [3-6], it gives rise to kinetic-scale, current filaments surrounded by toroidal magnetic fields, through which the charged particles are progressively isotropized $[3,7,8]$. This instability is widely thought to underpin the physics of relativistic outflows in powerful astrophysical objects (e.g., gamma-ray bursts, pulsar winds, active galactic nuclei), especially as the

\footnotetext{
*These authors have contributed equally to this work.

${ }^{\dagger}$ Corresponding author: gaurav.raj@ polytechnique.edu

${ }^{\ddagger}$ Corresponding author: olena.kononenko@ polytechnique.edu

${ }^{\S}$ Corresponding author: sebastien.corde@ polytechnique.edu
}

Published by the American Physical Society under the terms of the Creative Commons Attribution 4.0 International license. Further distribution of this work must maintain attribution to the author(s) and the published article's title, journal citation, and DOI. source of the collisionless shock waves held responsible for generating nonthermal high-energy particles and radiations [9-13]. Moreover, it is expected to operate in magnetic reconnection scenarios [14], and has been invoked as a possible generation mechanism for cosmological magnetic fields [15].

On the laboratory side, the CFI stands as a key process in intense laser-plasma interactions. In the case of overdense plasmas irradiated at relativistic laser intensities $\left(I_{0} \lambda_{0}^{2} \gtrsim\right.$ $10^{18} \mathrm{~W} \mathrm{~cm}^{-2} \mu \mathrm{m}^{2}$, where $I_{0}$ and $\lambda_{0}$ are the laser intensity and wavelength, respectively), it arises from the counterstreaming of the forward-directed, laser-accelerated fast electrons and the current-neutralizing, cold plasma electrons [16-19]. The resulting magnetic fluctuations may grow fast enough to cause significant scattering and deceleration of the fast electrons [20-23]. These effects are generally considered detrimental to fast-electron-based applications, e.g., the fast ignition approach to inertial confinement fusion [19] or target normal sheath ion acceleration [24-26]. Still, they can also be triggered purposefully in laboratory astrophysics experiments addressing the physics of collisionless shocks, whether involving relativistic laser-solid interactions [27,28], laser-driven interpenetrating plasma flows $[29,30]$, or electron beam-plasma interactions [31,32]. 


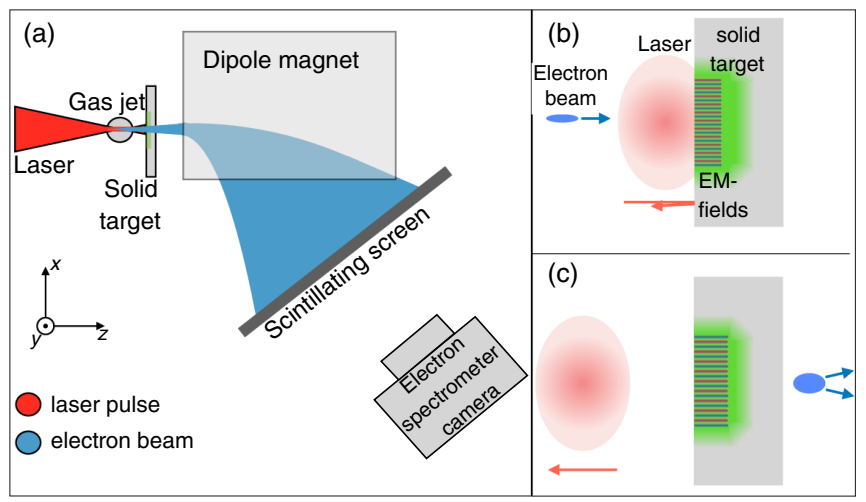

FIG. 1. Schematic of the experimental setup: (a) A laser pulse accelerates a relativistic electron beam from a supersonic gas jet, and is subsequently reflected off a solid foil target placed at the exit of the gas jet. The electron beam passes through the foil and is sent toward an electron spectrometer. (b), (c) When traveling across the foil, the beam electrons are scattered by the electromagnetic fluctuations driven by the laser pulse.

Experimental evidence for the development of the CFI in relativistic laser-driven plasmas has been mainly provided through characterization of the spatial profiles of the fast electron [33-36] or ion [24-26,37,38] beams exiting the target. In situ measurements of the magnetic-field fluctuations at the irradiated target surface have been performed using optical polarimetry $[39,40]$, yet this technique cannot access the volumetric distribution of the fields, and the data obtained so far could not capture their femtosecond timescale dynamics. Probing plasma electromagnetic fields by an ultrashort electron beam was previously exploited to image plasma wakefields in a laser wakefield accelerator (LWFA) [41] or large-scale inductively generated magnetic fields in target normal sheath acceleration [42].

In this paper, we demonstrate that the physics of the CFI developing at femtosecond timescales in the interaction of ultrashort, moderately relativistic laser pulses with solid targets can be explored by electron deflectometry using a 100-MeVrange probe electron bunch, produced by a laser wakefield accelerator [43-47]. Our measurements of the line-integrated magnetic field agree well with detailed three-dimensional (3D) fully relativistic particle-in-cell (PIC) simulations, and taken together, they indicate that the kT-level, submicronscale magnetic fluctuations indeed result from a Weibel-type CFI excited at the target surface. In our experimental setup, the laser pulse driving the LWFA is the same one that induces the electromagnetic fluctuations in a neighboring foil target (see Fig. 1). This ensures a well-controlled time delay between the electron bunch and the laser pump, and therefore probing of the field fluctuations a few tens of femtoseconds only after the on-target laser pulse maximum. Their line-integrated field strength is then inferred from the angular broadening induced upon the electron bunch and successfully compared to the numerical modeling.

\section{EXPERIMENTAL RESULTS}

The experiment was performed at Laboratoire d'Optique Appliquée with the "Salle Jaune" Ti:sapphire laser system, delivering laser pulses with 30-fs full width at half-maximum (FWHM) duration and up to 1.5-J energy on target. The laser pulse had a 810-nm central wavelength and was linearly polarized along the horizontal $x$ axis. Corrected using adaptive optics, it was focused at the entrance of a 3-mm exit diameter gas jet target by a $f / 16$ off-axis parabola to a $20-\mu \mathrm{m}$ FWHM spot size in vacuum, yielding a normalized peak vector potential of $a_{0} \simeq 1.5$ when accounting for the experimental intensity distribution in the focal plane. The supersonic gas jet used for the LWFA consisted of a mixture of $99 \%$ hydrogen and $1 \%$ nitrogen, enabling well-controlled electron acceleration through ionization injection [48-51]. Due to relativistic selffocusing and self-steepening in the LWFA stage, the laser field strength is expected to be enhanced to $a_{0} \gtrsim 3$ [52]. After exiting the gas jet, the laser pulse and the electron beam impinged on a thin Mylar or aluminium foil, located at a variable position along the propagation axis. The electron beam transmitted through the foil was characterized by an electron spectrometer comprising a 10-cm-long, 1.0-T dipole magnet deflecting electrons depending on their energy along the horizontal $x$ axis, and a scintillating screen imaged onto a 16-bit camera [see Fig. 1(a)]. The spectrometer also recorded angular information along the nondispersive vertical $y$ axis (perpendicular to laser polarization), but the large distance (about $35 \mathrm{~cm}$ ) between the foil and the scintillating screen prevented submicron-scale structures of the beam profile close to the target from being resolved.

The LWFA was operated in the highly nonlinear regime [53], and the electrons from the inner shells of the nitrogen dopant were ionized within the blowout cavity by the highintensity part of the laser pulse. This resulted in continuous injection as the laser propagated through the gas, and therefore in electron beams with a broad energy spectrum extending beyond $200 \mathrm{MeV}$ [Fig. 2(a) (top)], a 50-100 pC charge (above $100 \mathrm{MeV}$ ) and a 2-4 mrad FWHM divergence. The longitudinal separation between the electron beam and the laser pulse was on the order of the plasma wavelength $(\sim 10 \mu \mathrm{m}$ for an electron plasma density of $\sim 10^{19} \mathrm{~cm}^{-3}$ ). After exiting the gas jet, the peak intensity of the diffracting laser pulse decreased with the propagation distance.

Figure 2(a) displays typical electron energy-angle spectra recorded during the experiment. The top panel shows the reference spectrum from the LWFA (no solid target). When a 13$\mu \mathrm{m}$-thick Mylar foil is placed $0.42 \mathrm{~mm}$ from the gas jet exit, the beam divergence is significantly increased [Fig. 2(a), middle], as also demonstrated in Fig. 2(c). The beam divergence, which is seen to scale as $1 / \gamma(\gamma$ the electron relativistic factor), is strongly reduced when the jet-foil distance is increased to $2.61 \mathrm{~mm}$ [Fig. 2(a), bottom], corresponding to a decrease in the laser intensity on the solid target surface. Additional experimental data (see Supplemental Material [54]) shows that the beam divergence is still significantly affected at a jet-foil distance of $\sim 2.5 \mathrm{~mm}$ when using higher on-target laser energy $(2.5 \mathrm{~J})$. Multiple scattering of beam electrons in the foil due to elastic collisions cannot account for this behavior since it should cause a negligible increase in the divergence in 13- $\mu \mathrm{m}$-thick Mylar (scattering angle of $0.38 \mathrm{mrad}$ for 150 $\mathrm{MeV}$ electrons) and be independent of the foil position.

Figure 2(b) plots the variations in the electron beam divergence with the jet-foil distance (in the range from 0.25 


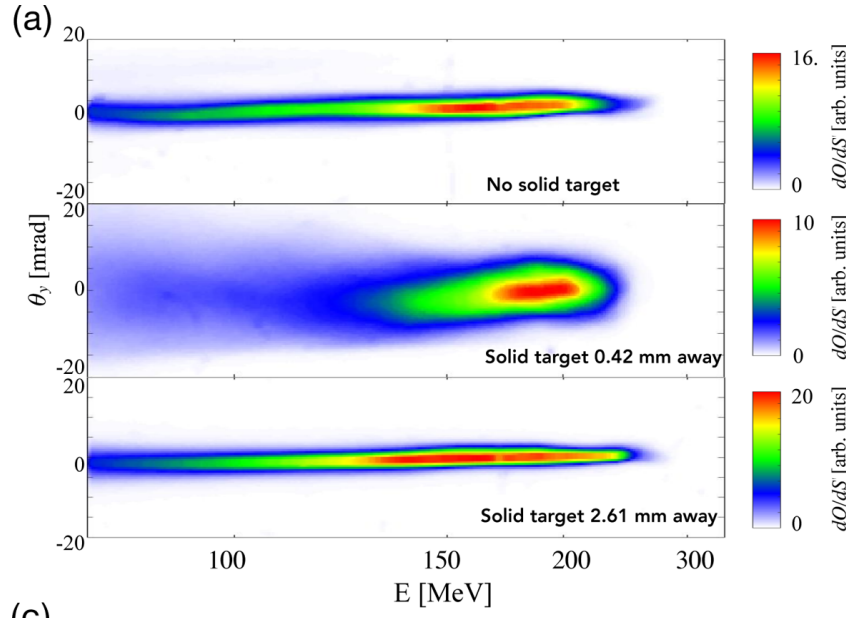

(c)

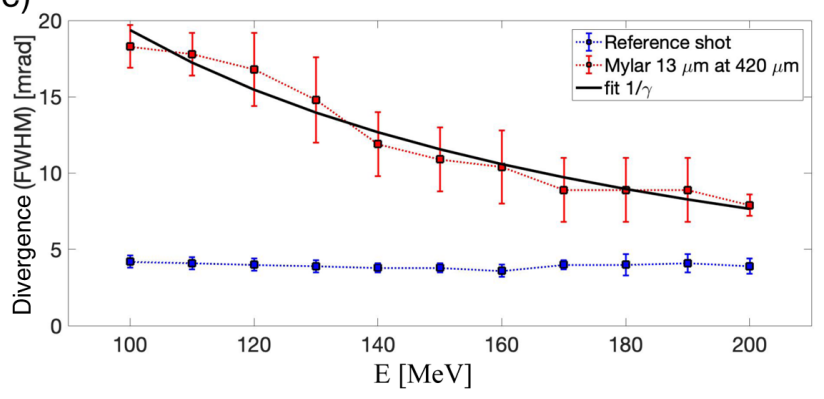

(b)

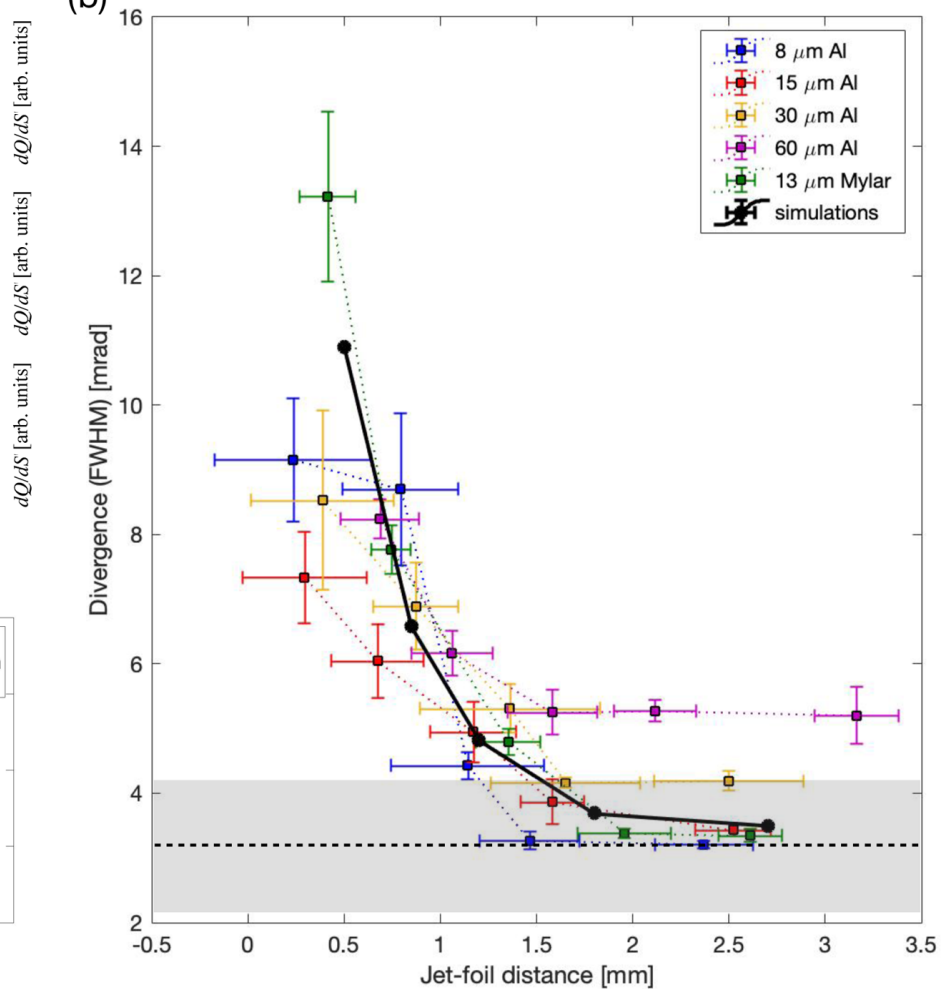

FIG. 2. Experimental results. (a) Typical electron spectra for the reference case (no solid target, top), and for distances of $0.42 \mathrm{~mm}$ (middle) and $2.61 \mathrm{~mm}$ (bottom) between the 13- $\mu$ m-thick Mylar foil and the gas jet exit. (b) Angular divergence (FWHM) of the 150-MeV beam electrons as a function of the distance between the gas jet exit and the solid foil: $8 \mu \mathrm{m} \mathrm{Al}$ (blue), $15 \mu \mathrm{m} \mathrm{Al} \mathrm{(red),} 30 \mu \mathrm{m} \mathrm{Al}$ (yellow), and $60 \mu \mathrm{m} \mathrm{Al}$ (magenta), as well as the 13- $\mu \mathrm{m}$-thick Mylar foil (green). The gray area represents the divergence of the reference shots (no solid target) together with its variation during the experiments. The black solid line corresponds to 3D PIC simulation results. (c) Angular divergence (FWHM) of the beam electrons as a function of their energy: reference shots (blue), shot of Fig. 2(a) middle (red, 13- $\mu$ m-thick Mylar foil at a distance of $0.42 \mathrm{~mm}$ ), and $1 / \gamma$ fit (black).

to $3.2 \mathrm{~mm})$ as measured with different targets (13- $\mu$ m-thick Mylar and 8- to 60- $\mu$ m-thick Al). Significant variation in the laser peak intensity at the solid target surface due to laser self-focusing and self-steepening in the laser wakefieldaccelerator results in relatively large shot-to-shot fluctuations. These could be strongly reduced with independent control of LWFA- and CFI-driving laser pulses. For each target type, the beam divergence is seen to decrease monotonically with the jet-foil distance. Increasing the Al foil thickness only entails detectable changes at large distances $(\gtrsim 1.5 \mathrm{~mm})$ due to stronger multiple scattering. These data indicate that the angular broadening of the electron beam takes place in the vicinity of the irradiated target surface. $3 \mathrm{D}$ simulation results, discussed below and plotted as a black solid line, provide a satisfactory match with the measurements.

As a result, our data provide a direct measurement of the integrated Lorentz force experienced by the beam in the solid foil, expressed as an equivalent line-integrated magnetic field

$$
B_{x, \text { int }}=\sqrt{\left\langle\left(\int B_{x} d z\right)^{2}\right\rangle_{n_{b}}},
$$

where the average is weighed by the transverse profile of the electron beam. This field induces a spread $\sigma_{p_{y}}=e B_{x, \text { int }}$ in the transverse momentum distribution of the electron beam, and therefore contributes to a total divergence $\theta_{y}^{2}=\theta_{y, \text { ref }}^{2}+\theta_{y, \mathrm{sc}}^{2}+$ $\theta_{y, \mathrm{~B}}^{2}$. Here, $\theta_{y, \text { ref }}$ is the original divergence of the LWFAgenerated beam, $\theta_{y, \mathrm{sc}}$ is the contribution from the multiple scattering, and $\theta_{y, \mathrm{~B}} \simeq \sigma_{p_{y}} / p_{z}=e c B_{x, \text { int }} / E$ is the contribution from the integrated equivalent magnetic field, with $E$ the electron energy. From the experimentally measured divergence, $\theta_{y}=13.23 \pm 1.31 \mathrm{mrad}$ (FWHM), of the $150-\mathrm{MeV}$ energy electrons passing through the 13- $\mu \mathrm{m}$-thick Mylar foil at a $0.42-\mathrm{mm}$ distance, one infers an integrated equivalent magnetic field of $B_{x, \text { int }}=2.70 \pm 0.39 \mathrm{kT} \mu \mathrm{m}$.

\section{SIMULATION RESULTS}

In order to identify the physical mechanism behind the electromagnetic field generation around the target surface, 2D and 3D PIC simulations have been performed using the code CALDER [55-58]. These fully relativistic simulations describe both the laser-foil and subsequent beam-plasma interactions, including the effects of binary Coulomb collisions, impact ionization, and field ionization. The laser is modeled as a planar wave with a Gaussian temporal profile and a 20-fs FWHM pulse duration. Its field strength on target is estimated to drop from $a_{0}=2.3$ to 0.7 when the jet-foil distance is increased from 0.5 to $2.7 \mathrm{~mm}$ (assuming 1 Joule of laser energy and $15 \mu \mathrm{m}$ FWHM spot size at the gas jet exit). 


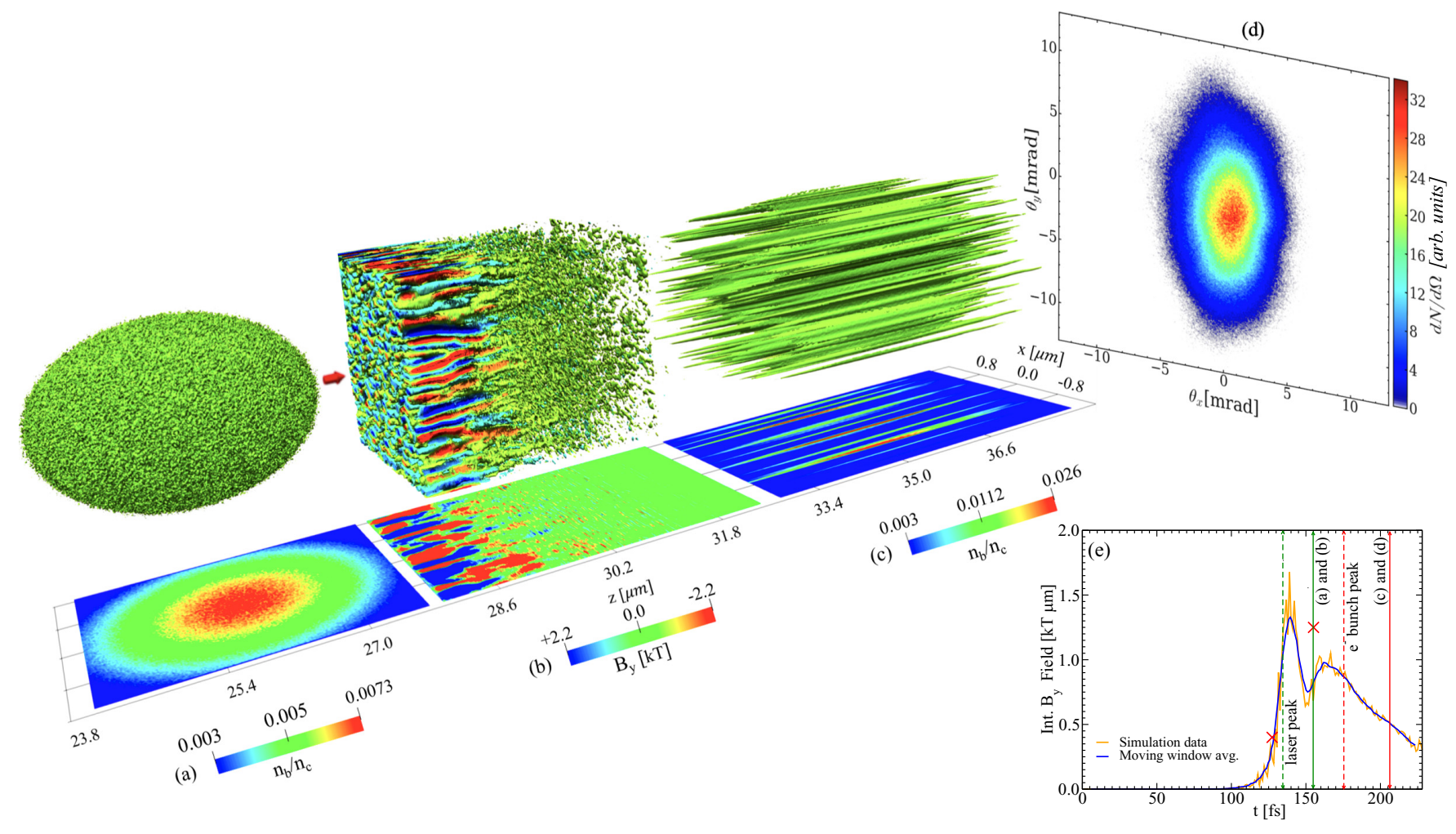

FIG. 3. 3D PIC simulation snapshots showing isosurfaces and slices at $y=0$ of (a) the electron bunch before entering the Al foil, (b) the $B_{y}$ component of the magnetic field generated due to laser-solid interaction, (c) the electron bunch after exiting the Al foil. The angular distribution of the final electron bunch is shown in (d), and (e) represents the temporal evolution of the $z$-integrated $B_{y}$ field obtained from $2 \mathrm{D}$ simulations using the same parameters as in the 3D simulation. In (e), the vertical lines indicate the time of arrival at the foil front surface of the peak of the laser pulse (dashed green) and of the electron beam (dashed red), and the time at which (a), (b) or (c), (d) snapshots are taken (respectively, green and red solid lines). Red crosses in (e) show the instantaneous values of $B_{y, \text { int }}$ from the 3D simulation, and the blue curve is a moving window average of the $2 \mathrm{D}$ simulation data (orange).

The electron beam is initialized with a $150-\mathrm{MeV}$ energy, a $1-\mu \mathrm{m}$ root-mean-square (RMS) transverse size, a $1.6-\mu \mathrm{m}$ RMS bunch length, a 50-pC total charge, and a $11-\mu \mathrm{m}$ peakto-peak separation with the laser pulse. The target consists of a $8-\mu \mathrm{m}$-thick, solid-density, neutral plasma of electrons and $\mathrm{Al}^{3+}$ ions. On its front side is added a linearly ramped preplasma of $0.8-\mu \mathrm{m}$ length to take account of an imperfect laser contrast (see Supplemental Material [54] for a discussion of the weak effect of the preplasma length on the resulting integrated $B$ field). The $3 \mathrm{D}$ domain size is $L_{x} \times L_{y} \times L_{z}=$ $2.1 \times 2.1 \times 45 \mu \mathrm{m}^{3}$ with a cell size in each direction of $\Delta x=$ $\Delta y=\Delta z=\lambda_{0} / 64$, while for the 2D simulations, the domain size is $L_{x} \times L_{z}=2.1 \times 45 \mu \mathrm{m}^{2}$ with a cell size $\Delta x=\Delta z=$ $\lambda_{0} / 64$. 50 macroparticles per cell for each species are used in all simulations.

Figure 3 shows results from the 3D PIC simulation for a 0.5 -mm jet-foil distance. While several mechanisms may give rise to strong electromagnetic fluctuations in the vicinity of the foil surface (e.g., parametric decay of laser-driven surface oscillations or Rayleigh-Taylor-type instability [59,60]), the Weibel-type CFI appears to be the dominant process under our experimental conditions (see Supplemental Material [54]). The resulting fluctuations, of mainly magnetic nature, exhibit a characteristic filamentary pattern with a $\sim 0.4-\mu \mathrm{m}$ transverse periodicity, and extending to a $\sim 1-\mu \mathrm{m}$ depth [Fig. 3(b)]. The time evolution of the $z$-integrated magnetic field during and after the laser irradiation is presented in Fig. 3(e), showing that the beam electrons experience fully grown magnetic fields as soon as they enter the target. Their $\left(\theta_{x}, \theta_{y}\right)$ angular distribution after transiting through the target is displayed in Fig. 3(d): the beam divergence along the vertical $(y)$ direction is measured to be $\theta_{y} \simeq 10 \mathrm{mrad}$ (FWHM), much larger than its initial value $(\simeq 0.1 \mathrm{mrad})$ in the simulation. Moreover, these magnetic deflections translate into strong transverse modulations in the beam profile [compare Figs. 3(a) and 3(c)]. The asymmetry between the horizontal $(x)$ and vertical $(y)$ divergences originates from the stronger laser-induced electron heating along the laser polarization axis $(x)$; this excites current modulations preferentially along the cold $(y)$ axis, hence leading to $B_{x, \text { int }}>B_{y, \text { int }}$ and to a larger vertical divergence. The effect of the head-on interaction between the reflected laser and the electron beam was found to be negligible when examining the beam angular distribution prior and after its collision with the reflected pulse, but before entering the solid target (see Supplemental Material [54]).

That a moderately relativistic laser pulse can generate kTrange magnetic fluctuations in a metal foil within a few $10 \mathrm{fs}$ only is not an obvious result. Interestingly, a self-consistent modeling of field ionization turns out to be necessary for a quantitative reproduction of the measurements. This was 


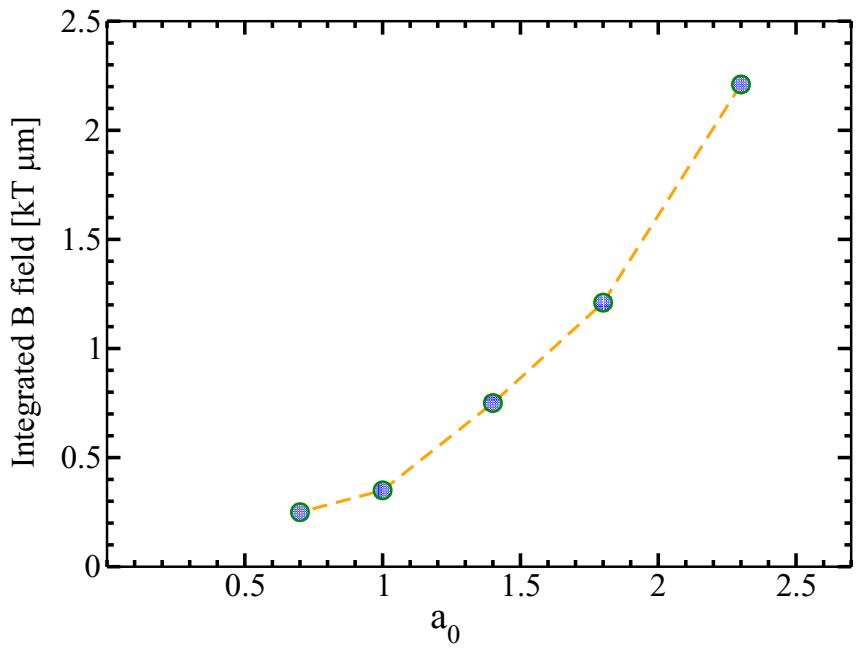

FIG. 4. Parameter scan using 3D PIC simulations, showing the variation in the line-integrated magnetic field $\left(B_{x, \text { int }}\right)$ as experienced by $150-\mathrm{MeV}$ electrons passing through a $8-\mu \mathrm{m}$-thick $\mathrm{Al}$ foil, with the laser strength $a_{0}$ (the change of which corresponds to a varying jet-foil distance in the experiment).

found by running additional 2D simulations in which either field [56] or collisional [57] ionization was deactivated. The major change arose when disabling field ionization, leading to a $\sim 5 \times$ drop in the $B$-field strength (see Supplemental Material [54]). Such mitigation of the CFI is ascribed to a decrease in both the background electron density and the forward momentum flux of the hot electrons driving the instability. This result is in line with a recent simulation study [61] which predicted the enhancement of the CFI by field ionization.

To further compare the simulation results with the experimental observations, and quantify the sensitivity of the CFIinduced magnetic fluctuations to the laser-driven intensity, we plot in Fig. 4 the results of a parametric scan where $a_{0}$ is varied from 2.3 to 0.7 , corresponding to a jet-foil distance ranging from 0.5 to $2.7 \mathrm{~mm}$. The integrated magnetic field experienced by the electron beam is seen to monotonically drop (down to $B_{x, \text { int }} \approx 0.25 \mathrm{kT} \mu \mathrm{m}$ at $a_{0}=0.7$ ) as the laser strength is reduced (or, equivalently, the foil is moved away from the gas jet).

\section{CONCLUSION}

To conclude, we have evidenced the generation of submicron-scale magnetic fields by the CFI excited during femtosecond ultraintense laser-solid interactions, and demonstrated, both experimentally and numerically, the potential of low-emittance LWFA-driven electron beams to probe these fields. Supported by PIC simulations, our measurements indicate that $B$ fields of $2.70 \pm 0.39 \mathrm{kT} \mu \mathrm{m}$ line-integrated strength build up at the front surface of a solid Al target irradiated by a $\sim 20 \mathrm{fs}, \sim 10^{19} \mathrm{~W} \mathrm{~cm}^{-2}$ laser pulse. Simulations show that the ultrafast CFI growth is furthered by field ionization. These results pave the way for measurements of the CFI dynamics at femtosecond timescales, by generating the probe electron beam from an auxiliary laser pulse with controlled delay. They are also of prime interest for staged plasma-based accelerators [62], including novel hybrid schemes, which aim to miniaturize beam-driven plasma wakefield accelerators (PWFA) $[63,64]$ and achieve unprecedented beam quality by using relativistic electron drive beams from a LWFA [65-70], and separating the LWFA and PWFA by a thin foil. This study highlights the need to mitigate the CFI (e.g., by depleting the laser pulse energy before it hits the solid target) so as to avoid degrading the quality of the electron beam driving the subsequent acceleration stage.

\section{ACKNOWLEDGMENTS}

This work was supported by the European Research Council (ERC) under the European Union's Horizon 2020 research and innovation programme (Miniature beam-driven Plasma Accelerators project, Grant Agreement No. 715807). H.D., A.D., M.F., M.F.G., and S.K. were supported by DFG (Deutsche Forschungsgemeinschaft), project name 'EXC 158: Munich-Centre for Advanced Photonics (MAP)' and project number 24819222 through the Cluster of Excellence $\mathrm{Mu}$ nich Centre for Advanced Photonics (MAP EXC 158). Numerical simulations were performed using HPC resources from PRACE (Partnership for Advanced Computing in Europe) (Grant No. 2017174175) and GENCI-TGCC (Grand Équipement National de Calcul Intensif) (Grants No. 2018A0040507594 and No. 2019-A0060510786) with the IRENE supercomputer.
[1] E. S. Weibel, Phys. Rev. Lett. 2, 83 (1959)

[2] B. D. Fried, Phys. Fluids 2, 337 (1959).

[3] R. C. Davidson, D. A. Hammer, I. Haber, and C. E. Wagner, Phys. Fluids 15, 317 (1972).

[4] A. Bret, L. Gremillet, and M. E. Dieckmann, Phys. Plasmas 17, 120501 (2010).

[5] A. Achterberg and J. Wiersma, Astron. Astrophys. 475, 1 (2007).

[6] C. Zhang, C.-K. Huang, K. A. Marsh, C. E. Clayton, W. B. Mori, and C. Joshi, Sci. Adv. 5, eaax4545 (2019).

[7] A. Achterberg, J. Wiersma, and C. A. Norman, Astron. Astrophys. 475, 19 (2007).
[8] C. Ruyer, L. Gremillet, A. Debayle, and G. Bonnaud, Phys. Plasmas 22, 032102 (2015).

[9] M. V. Medvedev and A. Loeb, Astrophys. J. 526, 697 (1999).

[10] A. Spitkovsky, Astrophys. J. Lett. 682, L5 (2008).

[11] T. N. Kato and H. Takabe, Astrophys. J. Lett. 681, L93 (2008).

[12] A. Marcowith, A. Bret, A. Bykov, M. E. Dieckman, L. O'C Drury, B. Lembège, M. Lemoine, G. Morlino, G. Murphy, G. Pelletier, I. Plotnikov, B. Reville, M. Riquelme, L. Sironi, and A. Stockem Novo, Rep. Prog. Phys. 79, 046901 (2016).

[13] M. Lemoine, L. Gremillet, G. Pelletier, and A. Vanthieghem, Phys. Rev. Lett. 123, 035101 (2019). 
[14] M. Swisdak, Y.-H. Liu, and J. F. Drake, Astrophys. J. 680, 999 (2008).

[15] R. Schlickeiser and P. K. Shukla, Astrophys. J. Lett. 599, L57 (2003).

[16] Y. Sentoku, K. Mima, S. Kojima, and H. Ruhl, Phys. Plasmas 7, 689 (2000).

[17] L. O. Silva, R. A. Fonseca, J. W. Tonge, W. B. Mori, and J. M. Dawson, Phys. Plasmas 9, 2458 (2002).

[18] C. Ren, M. Tzoufras, J. Tonge, W. B. Mori, F. S. Tsung, M. Fiore, R. A. Fonseca, L. O. Silva, J.-C. Adam, and A. Heron, Phys. Plasmas 13, 056308 (2006).

[19] A. P. L. Robinson, D. J. Strozzi, J. R. Davies, L. Gremillet, J. J. Honrubia, T. Johzaki, R. J. Kingham, M. Sherlock, and A. A. Solodov, Nucl. Fusion 54, 054003 (2014).

[20] Y. Sentoku, K. Mima, P. Kaw, and K. Nishikawa, Phys. Rev. Lett. 90, 155001 (2003).

[21] B. Chrisman, Y. Sentoku, and A. J. Kemp, Phys. Plasmas 15, 056309 (2008).

[22] J. C. Adam, A. Héron, and G. Laval, Phys. Rev. Lett. 97, 205006 (2006).

[23] A. Debayle, J. J. Honrubia, E. d'Humières, and V. T. Tikhonchuk, Phys. Rev. E 82, 036405 (2010).

[24] J. Fuchs, T. E. Cowan, P. Audebert, H. Ruhl, L. Gremillet, A. Kemp, M. Allen, A. Blazevic, J.-C. Gauthier, M. Geissel, M. Hegelich, S. Karsch, P. Parks, M. Roth, Y. Sentoku, R. Stephens, and E. M. Campbell, Phys. Rev. Lett. 91, 255002 (2003).

[25] S. Göde, C. Rödel, K. Zeil, R. Mishra, M. Gauthier, F.-E. Brack, T. Kluge, M. J. MacDonald, J. Metzkes, L. Obst, M. Rehwald, C. Ruyer, H.-P. Schlenvoigt, W. Schumaker, P. Sommer, T. E. Cowan, U. Schramm, S. Glenzer, and F. Fiuza, Phys. Rev. Lett. 118, 194801 (2017)

[26] G. G. Scott, C. M. Brenner, V. Bagnoud, R. J. Clarke, B. Gonzalez-Izquierdo, J. S. Green, R. I. Heathcote, H. W. Powell, D. R. Rusby, B. Zielbauer, P. McKenna, and D. Neely, New J. Phys. 19, 043010 (2017).

[27] F. Fiuza, R. A. Fonseca, J. Tonge, W. B. Mori, and L. O. Silva, Phys. Rev. Lett. 108, 235004 (2012).

[28] C. Ruyer, L. Gremillet, and G. Bonnaud, Phys. Plasmas 22, 082107 (2015).

[29] W. Fox, G. Fiksel, A. Bhattacharjee, P.-Y. Chang, K. Germaschewski, S. X. Hu, and P. M. Nilson, Phys. Rev. Lett. 111, 225002 (2013).

[30] C. M. Huntington, F. Fiuza, J. S. Ross, A. B. Zylstra, R. P. Drake, D. H. Froula, G. Gregori, N. L. Kugland, C. C. Kuranz, M. C. Levy, C. K. Li, J. Meinecke, T. Morita, R. Petrasso, C. Plechaty, B. A. Remington, D. D. Ryutov, Y. Sakawa, A. Spitkovsky, H. Takabe, and H.-S. Park, Nat. Phys. 11, 173 (2015).

[31] B. Allen, V. Yakimenko, M. Babzien, M. Fedurin, K. Kusche, and P. Muggli, Phys. Rev. Lett. 109, 185007 (2012).

[32] A. Benedetti, M. Tamburini, and C. H. Keitel, Nat. Photonics 12, 319 (2018).

[33] M. Tatarakis, F. N. Beg, E. L. Clark, A. E. Dangor, R. D. Edwards, R. G. Evans, T. J. Goldsack, K. W. D. Ledingham, P. A. Norreys, M. A. Sinclair, M.-S. Wei, M. Zepf, and K. Krushelnick, Phys. Rev. Lett. 90, 175001 (2003).

[34] M. S. Wei, F. N. Beg, E. L. Clark, A. E. Dangor, R. G. Evans, A. Gopal, K. W. D. Ledingham, P. McKenna, P. A. Norreys, M. Tatarakis, M. Zepf, and K. Krushelnick, Phys. Rev. E 70, 056412 (2004).
[35] R. Jung, J. Osterholz, K. Löwenbrück, S. Kiselev, G. Pretzler, A. Pukhov, O. Willi, S. Kar, M. Borghesi, W. Nazarov, S. Karsch, R. Clarke, and D. Neely, Phys. Rev. Lett. 94, 195001 (2005).

[36] M. Manclossi, J. J. Santos, D. Batani, J. Faure, A. Debayle, V. T. Tikhonchuk, and V. Malka, Phys. Rev. Lett. 96, 125002 (2006).

[37] J. Metzkes, T. Kluge, K. Zeil, M. Bussmann, S. D. Kraft, T. E. Cowan, and U. Schramm, New J. Phys. 16, 023008 (2014).

[38] M. King, N. Butler, R. Wilson, R. Capdessus, R. Gray, H. W. Powell, R. Dance, H. Padda, B. Gonzalez-Izquierdo, D. Rusby, N. Dover, G. S. Hicks, O. Ettlinger, C. Scullion, D. Carroll, Z. Najmudin, M. Borghesi, D. Neely, and P. Mckenna, High Power Laser Sci. Eng. 7, e14 (2019).

[39] S. Mondal, V. Narayanan, W. J. Ding, A. D. Lad, B. Hao, S. Ahmad, W. M. Wang, Z. M. Sheng, S. Sengupta, P. Kaw, A. Das, and G. R. Kumar, Proc. Natl. Acad. Sci. USA 109, 8011 (2012).

[40] G. Chatterjee, K. M. Schoeffler, P. Kumar Singh, A. Adak, A. D. Lad, S. Sengupta, P. Kaw, L. O. Silva, A. Das, and G. R. Kumar, Nat. Commun. 8, 15970 (2017).

[41] C. J. Zhang, J. F. Hua, Y. Wan, C.-H. Pai, B. Guo, J. Zhang, Y. Ma, F. Li, Y. P. Wu, H.-H. Chu, Y. Q. Gu, X. L. Xu, W. B. Mori, C. Joshi, J. Wang, and W. Lu, Phys. Rev. Lett. 119, 064801 (2017).

[42] W. Schumaker, N. Nakanii, C. McGuffey, C. Zulick, V. Chyvkov, F. Dollar, H. Habara, G. Kalintchenko, A. Maksimchuk, K. A. Tanaka, A. G. R. Thomas, V. Yanovsky, and K. Krushelnick, Phys. Rev. Lett. 110, 015003 (2013).

[43] T. Tajima and J. M. Dawson, Phys. Rev. Lett. 43, 267 (1979).

[44] J. Faure, Y. Glinec, A. Pukhov, S. Kiselev, S. Gordienko, E. Lefebvre, J.-P. Rousseau, F. Burgy, and V. Malka, Nature (London) 431, 541 (2004).

[45] C. G. R. Geddes, C. Toth, J. van Tilborg, E. Esarey, C. B. Schroeder, D. Bruhwiler, C. Nieter, J. Cary, and W. P. Leemans, Nature (London) 431, 538 (2004).

[46] S. P. D. Mangles, C. D. Murphy, Z. Najmudin, A. G. R. Thomas, J. L. Collier, A. E. Dangor, E. J. Divall, P. S. Foster, J. G. Gallacher, C. J. Hooker, D. A. Jaroszynski, A. J. Langley, W. B. Mori, P. A. Norreys, F. S. Tsung, R. Viskup, B. R. Walton, and Krushelnick, Nature (London) 431, 535 (2004).

[47] A. J. Gonsalves, K. Nakamura, J. Daniels, C. Benedetti, C. Pieronek, T. C. H. de Raadt, S. Steinke, J. H. Bin, S. S. Bulanov, J. van Tilborg, C. G. R. Geddes, C. B. Schroeder, C. Tóth, E. Esarey, K. Swanson, L. Fan-Chiang, G. Bagdasarov, N. Bobrova, V. Gasilov et al., Phys. Rev. Lett. 122, 084801 (2019).

[48] T. P. Rowlands-Rees, C. Kamperidis, S. Kneip, A. J. Gonsalves, S. P. D. Mangles, J. G. Gallacher, E. Brunetti, T. Ibbotson, C. D. Murphy, P. S. Foster, M. J. V. Streeter, F. Budde, P. A. Norreys, D. A. Jaroszynski, K. Krushelnick, Z. Najmudin, and S. M. Hooker, Phys. Rev. Lett. 100, 105005 (2008).

[49] A. Pak, K. A. Marsh, S. F. Martins, W. Lu, W. B. Mori, and C. Joshi, Phys. Rev. Lett. 104, 025003 (2010).

[50] C. McGuffey, A. G. R. Thomas, W. Schumaker, T. Matsuoka, V. Chvykov, F. J. Dollar, G. Kalintchenko, V. Yanovsky, A. Maksimchuk, K. Krushelnick, V. Y. Bychenkov, I. V. Glazyrin, and A. V. Karpeev, Phys. Rev. Lett. 104, 025004 (2010).

[51] A. Döpp, B. Mahieu, A. Lifschitz, C. Thaury, A. Doche, E. Guillaume, G. Grittani, O. Lundh, M. Hansson, J. Gautier, M. Kozlova, J. P. Goddet, P. Rousseau, A. Tafzi, V. Malka, 
A. Rousse, S. Corde, and K. Ta Phuoc, Light: Sci. Appl. 6, e17086 (2017).

[52] S. Corde, C. Thaury, A. Lifschitz, G. Lambert, K. Ta Phuoc, X. Davoine, R. Lehe, D. Douillet, A. Rousse, and V. Malka, Nat. Commun. 4, 1501 (2013).

[53] E. Esarey, C. B. Schroeder, and W. P. Leemans, Rev. Mod. Phys. 81, 1229 (2009).

[54] See Supplemental Material at http://link.aps.org/supplemental/ 10.1103/PhysRevResearch.2.023123 for additional experimental data with higher laser energy, and regarding preplasma length, magnetic-field generation mechanisms, and effects of reflected laser and field ionization.

[55] E. Lefebvre, N. Cochet, S. Fritzler, V. Malka, M.-M. Aléonard, J.-F. Chemin, S. Darbon, L. Disdier, J. Faure, A. Fedotoff, O. Landoas, G. Malka, V. Méot, P. Morel, M. Rabec LeGloahec, A. Rouyer, C. Rubbelynck, V. Tikhonchuk, R. Wrobel et al., Nucl. Fusion 43, 629 (2003).

[56] R. Nuter, L. Gremillet, E. Lefebvre, A. Lévy, T. Ceccotti, and P. Martin, Phys. Plasmas 18, 033107 (2011).

[57] F. Pérez, L. Gremillet, A. Decoster, M. Drouin, and E. Lefebvre, Phys. Plasmas 19, 083104 (2012).

[58] M. Lobet, E. d'Humières, M. Grech, C. Ruyer, X. Davoine, and L. Gremillet, J. Phys.: Conf. Ser. 688, 012058 (2016).

[59] A. Macchi, F. Cornolti, and F. Pegoraro, Phys. Plasmas 9, 1704 (2002).

[60] T. Kluge, J. Metzkes, K. Zeil, M. Bussmann, U. Schramm, and T. E. Cowan, Phys. Plasmas 22, 064502 (2015).

[61] Y. Lang, X. H. Yang, H. Xu, Z. Jin, and H. B. Zhuo, Plasma Phys. Control. Fusion 60, 075002 (2018).

[62] S. Steinke, J. van Tilborg, C. Benedetti, C. G. R. Geddes, C. B. Schroeder, J. Daniels, K. K. Swanson, A. Gonsalves, K. P. Nakamura, N. Matlis, B. Shaw, E. Esarey, and W. Leemans, Nature (London) 530, 190 (2016).
[63] P. Chen, J. M. Dawson, R. W. Huff, and T. Katsouleas, Phys. Rev. Lett. 54, 693 (1985).

[64] M. Litos, E. Adli, W. An, C. I. Clarke, C. E. Clayton, S. Corde, J. P. Delahaye, R. J. England, A. S. Fisher, J. Frederico, S. Gessner, S. Z. Green, M. J. Hogan, C. Joshi, W. Lu, K. A. Marsh, W. B. Mori, P. Muggli, N. Vafaei-Najafabadi, D. Walz et al., Nature (London) 515, 92 (2014).

[65] B. Hidding, T. Königstein, J. Osterholz, S. Karsch, O. Willi, and G. Pretzler, Phys. Rev. Lett. 104, 195002 (2010).

[66] S. Chou, J. Xu, K. Khrennikov, D. E. Cardenas, J. Wenz, M. Heigoldt, L. Hofmann, L. Veisz, and S. Karsch, Phys. Rev. Lett. 117, 144801 (2016).

[67] J. Ferri, S. Corde, A. Döpp, A. Lifschitz, A. Doche, C. Thaury, K. Ta Phuoc, B. Mahieu, I. A. Andriyash, V. Malka, and X. Davoine, Phys. Rev. Lett. 120, 254802 (2018).

[68] M. F. Gilljohann, H. Ding, A. Döpp, J. Götzfried, S. Schindler, G. Schilling, S. Corde, A. Debus, T. Heinemann, B. Hidding, S. M. Hooker, A. Irman, O. Kononenko, T. Kurz, A. Martinez de la Ossa, U. Schramm, and S. Karsch, Phys. Rev. X 9, 011046 (2019).

[69] A. Martinez de la Ossa, R. W. Assmann, M. Bussmann, S. Corde, J. P. Couperus Cabadag, A. Debus, A. Döpp, A. Ferran Pousa, M. F. Gilljohann, T. Heinemann, B. Hidding, A. Irman, S. Karsch, O. Kononenko, T. Kurz, J. Osterhoff, R. Pausch, S. Schöbel, and U. Schramm, Philos. Trans. R. Soc. A 377, 20180175 (2019).

[70] T. Kurz, T. Heinemann, M. F. Gilljohann, Y. Y. Chang, J. P. C. Cabadağ, A. Debus, O. Kononenko, R. Pausch, S. Schöbel, R. W. Assmann, M. Bussmann, H. Ding, J. Götzfried, A. Köhler, G. Raj, S. Schindler, K. Steiniger, O. Zarini, S. Corde, A. Döpp et al., arXiv:1909.06676. 